\title{
Toy Poodle
}

National Cancer Institute

\section{Source}

National Cancer Institute. Toy Poodle. NCI Thesaurus. Code C53861.

The Toy Poodle is a small poodle with a height up to 10 inches and a weight of 6-9 pounds. 